\title{
A non-linear model of rubber shear springs validated by experiments
}

\author{
Sanpeng Gong ${ }^{* * *}$, Sebastian Oberst ${ }^{* *}$ and Xinwen Wang ${ }^{*}$ \\ *School of Chemical and Environmental Engineering, China University of Mining and Technology, Beijing, China \\ ${ }^{*}$ Centre for Audio, Acoustics and Vibration, University of Technology Sydney, Australia
}

\begin{abstract}
Vibrating flip-flow screens provide an effective solution for screening highly viscous or fine materials. However, yet, only linear theory has been applied to their design. Yet, to understand deficiencies and to improve performance an accurate model especially of the rubber shear springs equipped in screen frames is critical for its dynamics to predict e.g. frequency- and amplitude-dependent behaviour. In this paper, the amplitude dependency of the rubber shear spring is represented by employing a friction model in which parameters are fitted to an affine function rather constant values used for the classic Berg's model; the fractional derivative model is used to describe its frequency dependency and compared to conventional dashpot and Maxwell models with its elasticity being represented by a nonlinear spring. The experimentally validated results indicate that the proposed model with a nonlinear spring, friction and fractional derivative model is able to more accurately describe the dynamic characteristics of a rubber shear spring compared with other models.
\end{abstract}

\section{Introduction}

Vibrating flip-flow screens (VFFS) play an important role in the dry screening of wet and fine materials such as gold deposited rocks, iron or coal ore. As one of the key components, the rubber shear spring exhibits frequencyand amplitude-dependent behaviour that largely affects the vibration characteristics of VFFS and which is responsible for a screen's performance [1,2]. To date, researchers have mostly investigated the influence of factors such as frequency and amplitude on rubber material properties. As frequency dependence the increase in frequency as a response of an increasing stiffness prior to viscous effects within the rubber material is predicted. The most widely used model when considering frequency-dependent behaviour is the Kelvin-Voight model in which a linear spring is installed in parallel with a viscous dashpot [3]. Yet, even though widely used, this model overestimates both stiffness and damping in the higher frequency regimes [4]. Placing a spring serially to a viscous dashpot, a three-parameter Maxwell model can be obtained [5], which underestimates the damping but better approximates the dynamic stiffness at high frequencies. To improve the prediction of the frequencydependent dynamics a fractional derivative model has been used [6,7]. For better describing the amplitudedependent behaviour of rubber properties, Berg [5] presented a smooth friction model with two constants which were extracted using large amplitude harmonic excitation at low frequencies; this simple analytical model better approximates the smoothness of measured curves than e.g. the stick-slip component model [6]. The parameters are obtained under a harmonic, large amplitude excitation at low frequency. However, this friction model underestimates stiffness and damping relative to the measurements when the excitation amplitude is small. Zhu [8] enhanced Berg's friction model through parameters estimation using statistical methods and remedied the effect the friction force had on the amplitude-dependent behaviour.

This paper presents a novel rubber shear spring model composed of an elastic, an amplitude- and a frequencydependent model. For improved accuracy a non-linear spring replaces the linear spring $[5,6]$. The friction model uses fitted parameters to an affine function by maintaining Berg's smooth friction model philosophy, and the viscoelastic property is described as a fractional derivative model using only two parameters. As validated by experiments, the new model enhances the prediction of the amplitude-dependent behaviour of rubber shear springs. Further, the frequency-dependent behaviour is also more accurately described using the fractional derivative model compared to a dashpot or a Maxwell model.

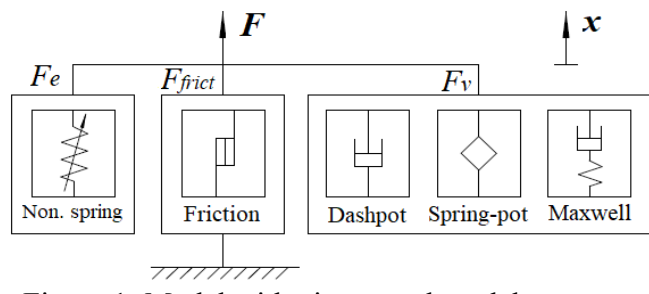

Figure 1: Model with viscous submodels.

\section{Model development}

Based on the specific experimental results, a phenomenological model of the rubber spring should be capable in representing the properties of the spring's nonlinear stiffness, its hysteresis as well as its amplitude- and frequency-dependency. An elastic sub-model represents the static nonlinear stiffness characteristics, the friction model accounts for the hysteresis and the amplitude dependency, and the viscous model is responsible for the frequency-dependent dynamics (Figure1).

Elastic model. The elastic model consists of a nonlinear relationship between the displacement $x=$ $x_{0} \sin (\omega t)$ and the resulting elastic force $F_{e}=K_{e}\left(a+b x_{0}\right) x_{0}$ with $x_{0}$ being the excitation amplitude. Stiffness $K_{e}$, and the non-dimensional parameters of $a$ and $b$ are obtained through experiments.

Friction model. The friction model uses experimental parameters fitted to an affine formula (constant and linear part) maintaining Berg's prerequisite of smoothness. The relationship between the displacement $x=x_{0} \sin (\omega t)$ and the corresponding friction force $F_{\text {frict }}$ is described as $F_{\text {frict }}=F_{f_{s}}$ for $x=x_{s}$; $F_{\text {frict }}=F_{f s}+\left(x-x_{s}\right)\left(F_{f \max }-F_{f s}\right) /\left(x_{2}(1-\varepsilon)+\left(x-x_{s}\right)\right)$ for $x>x_{s}$; and $F_{\text {frict }}=F_{f s}+(x-$ 
$\left.x_{s}\right)\left(F_{f \max }+F_{f s}\right) /\left(x_{2}(1+\varepsilon)-\left(x-x_{s}\right)\right)$ for $x<x_{s}$. Here $x_{s}$ and $F_{f s}$ are the reference displacement and force, and $F_{f \max }$ and $x_{2}$ are the maximum friction shear force and the displacement at half of $F_{f \max }$, which vary under different excitation amplitude $x_{0} ; F_{f \max }=\left(c+d x_{0}\right)$ and $x_{2}=\left(e+f x_{0}\right)$, where $c, d, e$ and $f$ are non-dimensional parameters which are obtained through measurements and $\varepsilon=F_{f s} / F_{f m a x}$ ranging between -1 and 1 .

Viscous model. Commonly the viscous properties are modelled using a linear viscous dashpot with damping parameter $c_{d}$ and viscous force $F_{v d}=c_{d} \dot{x}$. Another viscous model is the so-called Maxwell module which inherently exhibits compatible dynamic behaviour. The viscoelastic force amplitude is expressed as $F_{v 0}=$ $\omega C_{M} x_{0} / \sqrt{1+\left(\omega C_{M} / K_{M}\right)^{2}}$, where $K_{M}, C_{M}$ are the stiffness of the linear spring and the damping of the linear damper, respectively, with $\omega$ being the circular excitation frequency. The fractional derivative module, generalises the operation of differentiation to non-integer orders, and its viscoelastic force is defined as $F_{v f}=b \frac{d^{\alpha} x}{d t^{\alpha}}$. Here, $\alpha(0<\alpha<1)$ and $b(b>0)$ are model constants which represent the order of the timederivative and the amplitude of the viscoelastic force.

\section{Results and discussion}

Amplitude-dependent results of experiment and simulation of the newly developed model, as well as the classic Berg model [5], are illustrated in Figure 2(a, b). The amplitude-dependence as observed in the experiments matches well with the new model behaviour. With an initial increase of the amplitude, the measured stiffness decreases while the damping increases, before it starts decreasing. Plotting the frequency response of the experiments and for the models produces Figure 2(c) and (d) which shows model 1 (nonlinear spring+ friction + dashpot); model 2 (nonlinear spring + friction + Maxwell model); and the newly proposed model (nonlinear spring + friction + fractional derivative model). Model 1 performs poorly in approximating the measured stiffness and the damping. Model 2 results in a better fit of the stiffness than model 1 , but the damping is far off from the experimental results. The newly proposed model shows the best fit of both, the measured stiffness and the damping compared with model 1 and model 2.
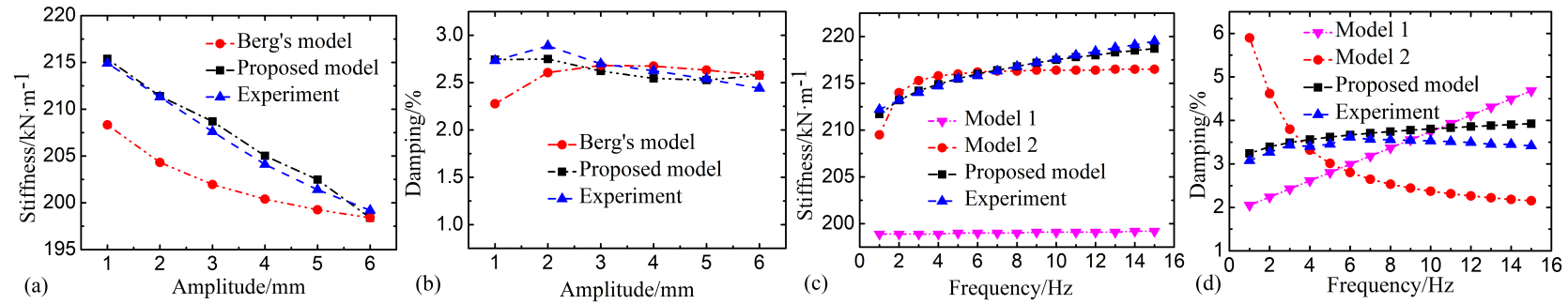

Figure 2: Stiffness, and damping versus (a), (b) excitation amplitude and versus (c), (d) excitation frequency

\section{Conclusion}

In this study we developed a nonlinear model of a rubber shear spring to illustrate the dependency of the rubber friction to the amplitude and the frequency-dependency as consequence of the viscoelastic properties of the rubber. This model is validated experimentally using quasi-static and harmonic tests. The elastic element in the proposed model is represented by a nonlinear spring, with its parameters being fitted to an affine function by maintaining smooth friction. The fractional derivative model is then used to describe the viscoelastic property of the rubber shear spring. Several models for the investigated rubber spring are compared with experimental results under quasi-static and harmonic excitation. The newly proposed model is capable to describe the amplitude and frequency-dependency more accurately than traditional models. The research work presented here will form the foundation to study the dynamics of VFFS equipped with rubber shear springs to improve performance and processing capacity.

\section{References}

[1] Xiong X., Niu L., Gu C., Wang Y. (2017) Vibration characteristics of an inclined flip-flow screen panel in banana flip-flow screens. J. Sound Vib. 411:108-128.

[2] Gong, S., Wang, X., Oberst, S. (2018) Non-linear analysis of vibrating flip-flow screens. Paper No. ICDME2018-112. ICDME 2018, Melbourne, Australia, July 16-18, 2018.

[3] Knothe K.L., Grassie, S.L. (1993) Modelling of Railway Track and Vehicle/Track Interaction at High Frequencies. Veh. Syst. Dyn. 22: 209-262.

[4] Babitsky, V.I., Veprik, A.M. (1998) Universal Bumpered Vibration Isolator for Severe Environment. J. Sound and Vib. 218: $269-292$.

[5] Berg M. (1998) A Non-Linear Rubber Spring Model for Rail Vehicle Dynamics Analysis. Veh. Syst. Dyn. 30:197-212.

[6] Sjöberg M. (2003) Nonlinear Isolator Dynamic at Finite Deformations: An Effective Hyperelastic, Fractional Derivative, Generalized Friction Model. Non. Dyna. 33: 323-336.

[7] Sedlaczek K., Dronka S., Rauh J. (2011) Advanced Modular Modelling of Rubber Bushing for Vehicle Simulations. Veh. Syst. Dyn. 49: 741-759.

[8] Zhu H., Yang J., Zhang Y., Feng X. (2017) A Novel Air Spring Dynamic Model with Pneumatic Thermodynamics, Effective Friction and Viscoelastic Damping. J. Sound Vib. 408:87-104. 